\title{
Estimation of Hidden Neuron Requisite for Predictive Conversion from Sign Language to a Formal Language
}

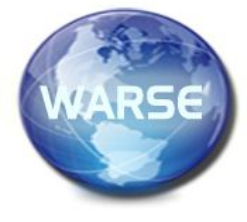

\author{
Sowjanya $M \mathbf{N}^{1}$, Thimmaraju $S \mathbf{N}^{2}$ \\ ${ }^{1}$ Assistant Professor, Department of MCA, \\ VTU-RRC, Mysuru, Karnataka,India \\ sowjanya12.mn@gmail.com \\ ${ }^{2}$ Professor. Department of MCA, \\ VTU-RRC, Belagavi, Karnataka, India
}

\begin{abstract}
Using sign language for communication has existed since millennia and has evolved over the ages to support the hearing impaired to a great extent. There have been numerous wonderful inventions and innovations aimed at improving the living conidtions of the hearing or speech impaired using technology which was contemporary. However, till date there exists no standard that can be shown as a single universal sign language translation system. This is partially due to the research happening in small pockets and lack of technology penetration in some countries. Largely the efforts to design translation are concentrated on Americal Sign Language (ASL) and Thai Sign Language (TSL). Other than the two, Indonasian Sign Language (SIBI) is the front runner in standardising a translation system. In our previous paper we had attempted to develop an ANN based translator for ASL. The system performed as per expectations. However, since the number of hidden neurons was taken as a variable, the estimation still remained a challenge. In this paper, we have developed a system to estimate the number of hidden neurons required for the accurate translation of ASL.
\end{abstract}

Key words: ANN, ASL, Estimation, Hidden Neurons, Sign Language

\section{INTRODUCTION}

In sign languages, any symbol takes a while for the viewer or a machine to translate. Most systems use multi-processing models or network computing to achieve faster results. However, the question remains that what should be the method used and what are the parameters needed to maintain consistency in the result of the translation. In our previous work, we had developed an ANN based translator for American Sign Language (ASL). In the translation system we had utilized a variable hidden neuron model. The challenge still remained as to how the number of hidden neurons could be optimized. In this paper we present the machine learning model using analysis of variation (AnoVa) to estimate the number of neurons needed for improved and consistent accuracy of the translation system. The paper presents a review of literature, a methodology, experimentation details and data and finally a logical conclusion that can open up new pathways into further research.

\section{LITERATURE REVIEW}

Thai translation system using upright speed-up robust feature and c-means clustering" [1] and "Thai translation system using upright speed-up robust feature and dynamic time warping" [2], have shown clearly that for a sign language that has symbols and spellings through gestures, two separate translation systems are required.

"Computer translation system for hearing impaired users" [3], have shown the peculiarities of the Russian sign language and illustrated that a single translation system cannot be developed for a language that has so many features.

"Research on Chinese-Japanese Translation System" [4], illustrated that all sign languages of the world are different from one another. With the increasing of economic and cultural exchanges between China and Japan, more and more hearing/ speech impaired people are working across borders which require a far more stable translator between the two sign languages.

"Plenary talk II: Recent developments in recognition systems" [5] has shown that Automated translation systems for sign languages are the need of the hour for the hearing and speech impaired as they aid the communication in a world where communication is the key to growth.

"Sign language localization: Learning to eliminate language dialects" [6] presented that any translation system from sign language to formal language has to work in two phases namely mapping and prediction. It is the first to refer to an ANN for translation.

"Toward transcription model in XML for Processing gloss annotation system" [7] illustrated that Representation of a sign language can be then converted into an animation using an avatar so that the rendition becomes interactive.

"Evaluating a System for Deaf People"[8] have shown a three tier translation system could be developed into a standard using example, rule based classifier and a statistical prediction.

"Vision-based translation device"[9] developed a system to translate single handed gestures of English alphabet and 
Sowjanya M N et al., International Journal of Emerging Trends in Engineering Research, 9(5), May 2021, 545 - 548

numbers into their formal values using image processing, to produce consistent results that can lead to a standardized system.

[10], [11], [12], [13], [16], [18] and [23] developed devices such as smart gloves, kinekt ${ }^{\mathrm{TM}}$ based gesture tracking and avatar based translation for various sign languages. Each system had its own advantages and disadvantages being a localized approach.

[14] Sign Language synthesis using hand motion acquisition and "Mobile motion gesture design for deaf people" [19]illustrated that motion capture could play a key role in sign language translation. As the transition from one sign to another is quite fluidic in humans, machines need to be trained to handle videos rather than images.

[15] and [17] have shown that text analysis and semantics when used in sign language translation, produce results that can be used in any colloquial sign language as well as rehabilitation of speech or hearing impaired people.

[20] has effectively proven that a classification of the formal language equivalent into its respective part of speech is a major task in sign language translation. This marks a future challenge in our proposed work.

[21] and [22] have conducted an extensive analysis of the Arabic sign language from a machine learning based translation system perspective. Their results provide insight into how one can incorporate the Arabic sign language into a standard translation system.

[24] shows that ANN based translators are the best suited systems for any sign language irrespective of the number of symbols.

In [25] and [26] we have shown that "Using ANN to predict formal language word from gesture is still in research stage and so far even though there are many Android OS based applications to assist the hearing impaired, we have still a far way to go for having an end-to-end solution"

In [27] the work carried out focuses on the application of $\mathrm{CNN}$ as deep learning for sign language translation. Its results are quite compelling and can be reproduced in an experimental set up.

\section{MethodolgY}

To achieve the set goals, the data generated from [25] was needed. However, it was not sufficient. We ran the experiment multiple times using various number of hidden neurons and tabulated the data for further comparison. The details of the experiment are given in the next section.

\section{EXPERIMENTATION}

The data generated for the ASL translation was with 4 neurons in the hidden layer. The same was run with 5, 6 and 7 hidden neurons respectively. The data generated with the final accuracy is shown in Table 1. The accuracies are plotted against the word count in Figures 1 through 4 respectively.
The final accuracies are plotted ina stacked graph for discernible difference visibility in figure 5. From the graph in Figure 5. It is visibly clear that the accuracy appears to be high. However on closer observation it can be seen that, the stability in accuracy is more when the number of neurons is set at 5 . Hence it can be stated that the proposed translation system works best when the number of hidden neurons is 5 in single layer.

Table 1. Final accuracies of the variable hidden neuron based translation

\begin{tabular}{|r|c|c|c|c|}
\hline & \multicolumn{4}{|c|}{ Hidden Neurons } \\
\hline Count & 4 & 5 & 6 & 7 \\
\hline 10 & 0.999153 & 0.999153 & 0.999153 & 0.999153 \\
\hline 13 & 0.963324 & 0.963328 & 0.963332 & 0.963336 \\
\hline 18 & 0.982165 & 0.982165 & 0.982166 & 0.982166 \\
\hline 30 & 0.986482 & 0.986483 & 0.986483 & 0.986483 \\
\hline 48 & 0.990986 & 0.990986 & 0.990986 & 0.990986 \\
\hline 50 & 0.983482 & 0.983482 & 0.983483 & 0.983483 \\
\hline 161 & 0.986595 & 0.986595 & 0.986596 & 0.986596 \\
\hline 326 & 0.989013 & 0.989013 & 0.989013 & 0.989013 \\
\hline 338 & 0.979844 & 0.979845 & 0.979846 & 0.979846 \\
\hline 380 & 0.983257 & 0.983257 & 0.983258 & 0.983258 \\
\hline 863 & 0.98811 & 0.98811 & 0.98811 & 0.98811 \\
\hline 1291 & 0.989008 & 0.989008 & 0.989009 & 0.989009 \\
\hline 1478 & 0.98798 & 0.98798 & 0.98798 & 0.98798 \\
\hline 1691 & 0.989155 & 0.989155 & 0.989155 & 0.989155 \\
\hline 2430 & 0.989296 & 0.989296 & 0.989296 & 0.989296 \\
\hline 3272 & 0.990826 & 0.990826 & 0.990826 & 0.990826 \\
\hline 3655 & 0.989982 & 0.989982 & 0.989982 & 0.989982 \\
\hline
\end{tabular}

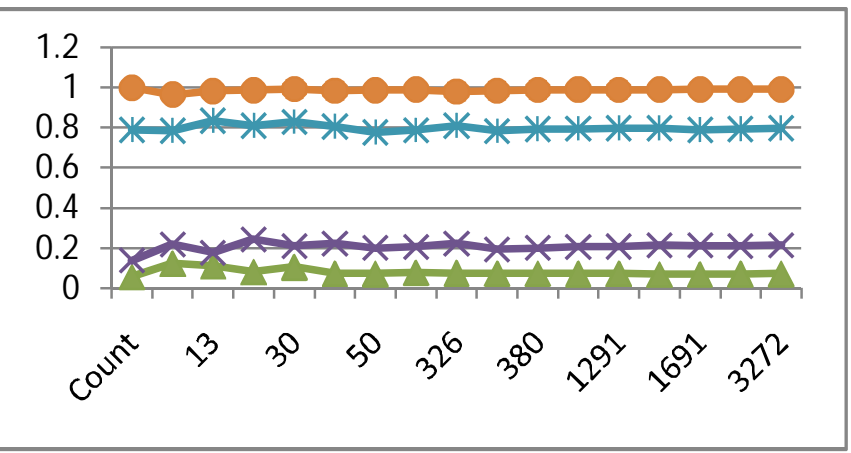

Figure 1. Accuracy with 4 hidden neurons 


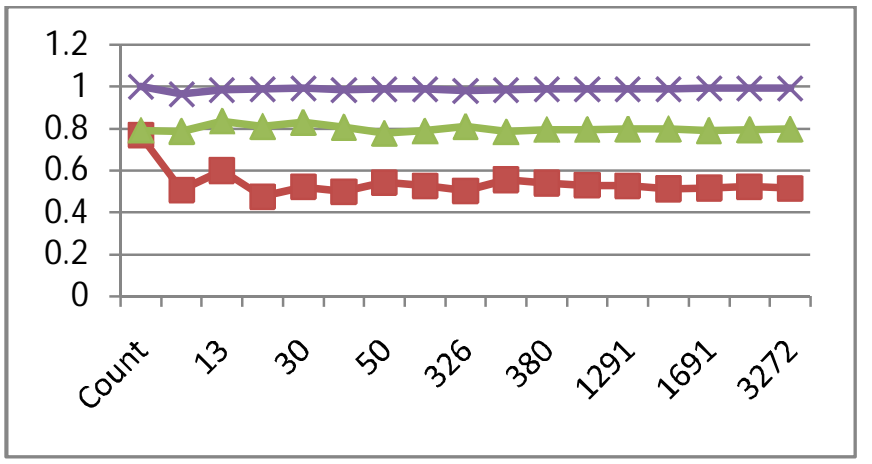

Figure 2. Accuracy with 5 hidden neurons

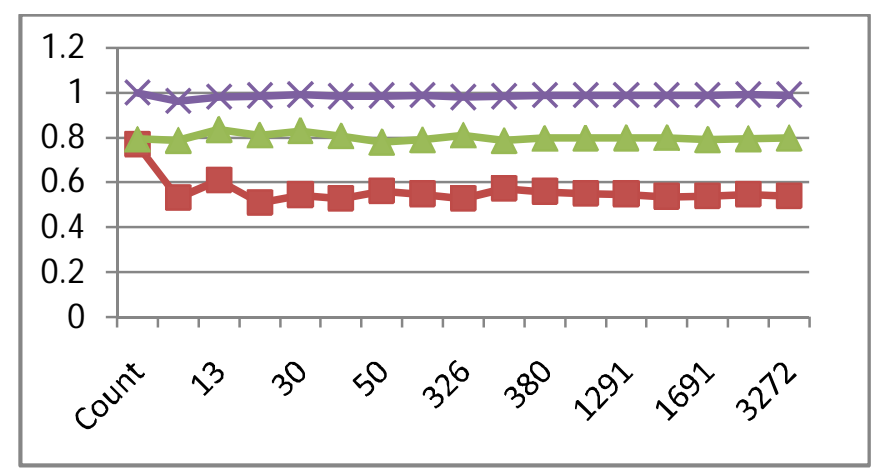

Figure 3. Accuracy with 6 hidden neurons

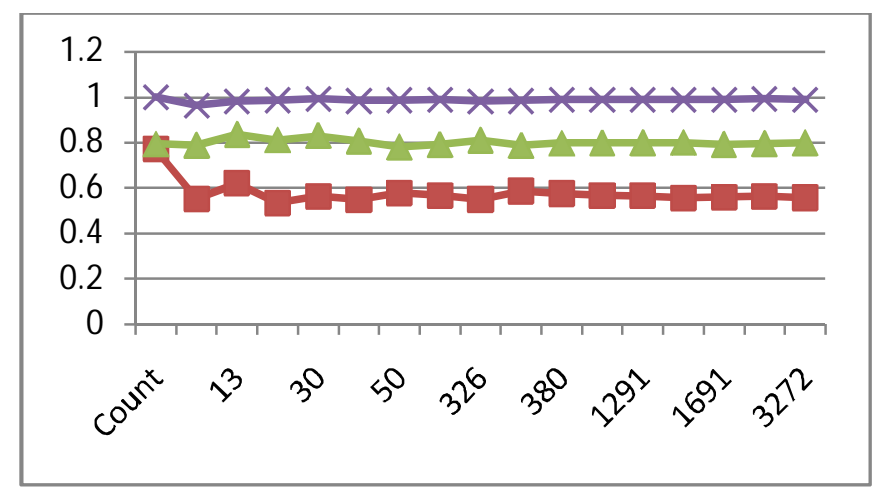

Figure 4. Accuracy with 7 hidden neurons

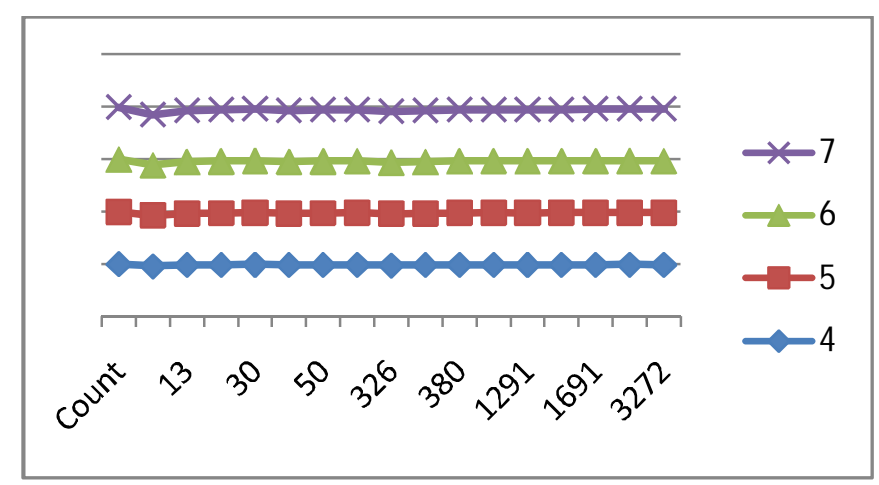

Figure 5. The final accuracies stacked to show the difference

\section{CONCLUSION}

To conclude, we can say that automated sign language translation systems are the future of bridging the gap between the normal world and the hearing/speech impaired. Towards this future, ANN based variable hidden neuron model as presented in our earlier work is a positive step and in this paper we have made a successful attempt to standardise the system.

\section{REFERENCES}

1. Chanda, P. ; Comput. Eng. Dept., Chiang Mai Univ., Chiang Mai, Thailand ; Auephanwiriyakul, S. ; TheeraUmpon, N. "Thai sign language translation system using upright speed-up robust feature and c-means clustering”, Fuzzy Systems (FUZZ-IEEE), 2012 IEEE International Conference, 2012.

2. Chanda, P. ; Comput. Eng. Dept., Chiang Mai Univ., Chiang Mai, Thailand ; Auephanwiriyakul, S. ; TheeraUmpon, N.,"Thai sign language translation system using upright speed-up robust feature and dynamic time warping",Computer Science and Automation Engineering (CSAE), 2012 IEEE International Conference on (Volume:2 ),2012

3. Grif, Mikhail G. ; Department of Automatic Control Systems, Novosibirsk State Technical University, Novosibirsk, Russia ; Korolkova, Olga O. ;Demyanenko, Yelena A. ; Tsoy, Evgeniy B., "Computer sign language translation system for hearing impaired users", Strategic Technology (IFOST), 2012 7th International Forum on , 2012

4. Zhen Liu ; Grad. Sch. of Eng., Nagasaki Inst. of Appl. Sci., Nagasaki, Japan ; Xiaomei Zhang; Kato, J.,"Research on Chinese-Japanese Sign Language Translation System", Frontier of Computer Science and Technology (FCST), 2010 Fifth International Conference on, 2010.

5. Tolba, M.F. ; Fac. of Comput. \& Inf. Sci., Ain Shams Univ., Cairo, Egypt, "Plenary talk II: Recent developments in sign language recognition systems", Computer Engineering \& Systems (ICCES), 2013 8th International Conference on ,2013

6. Tariq, M. ; Dept. of Comput. Sci., Kinnaird Coll. for Women, Lahore, Pakistan ; Iqbal,A. ; Zahid,A. ; etal "Sign language localization: Learning to eliminate language dialects", Multitopic Conference (INMIC), 2012 15th International,2012

7. Othman, Achraf ; Research Laboratory of Technologies of Information and Communication \& Electrical Engineering LaTICE-GE, Tunis National Higher School of Engineering (ENSIT), 5, Avenue Taha Hussein, B.P. 56, Bab Menara, 1008, University of TUNIS, Tunisia ; Hamdoun, Raouia, "Toward a new transcription model in XML for Sign Language Processing based on gloss annotation system", Information and Communication Technology and Accessibility (ICTA), 2013 Fourth International Conference on, 2013. 
Sowjanya M N et al., International Journal of Emerging Trends in Engineering Research, 9(5), May 2021, 545 - 548

8. Lopez-Ludena, V.; Grupo de Tecnol. del Habla, Univ. Politec. de Madrid, Madrid, Spain ; San-Segundo, R. ; Martin, R. ; Sanchez, D., "Evaluating a Speech Communication System for Deaf People", Latin America Transactions, IEEE (Revista IEEE America Latina) (Volume:9, Issue: 4 ), 2011

9. Madhuri, Y.; Dept. of Biomed. Eng., SRM Univ., Kattankulathur, India ; Anitha, G. ; Anburajan, M.,"Vision-based sign language translation device", Information Communication and Embedded Systems (ICICES), 2013 International Conference on, 2013

10. Trottier-Lapointe, W. ; PolyProject, Tech. Soc. of Ecole Polytech. de Montreal, Montréal, QC, Canada ; Majeau, L. ; El-Iraki, Y.; Loranger, S. "Signal processing for low cost optical data glove", Information Science, Signal Processing and their Applications (ISSPA), 2012 11th International Conference on, 2012

11. Verma, H.V.; Dept. of Comput. Sc. \& Eng., Jaypee Inst. of Inf. Technol., Noida, India ; Aggarwal, E. ; Chandra, S. ,"Gesture recognition using kinect for sign language translation", Image Information Processing (ICIIP), 2013 IEEE Second International Conference on, 2013

12. Lombardo, V.; Dipt. di Inf., Univ. degli Studi di Torino, Torino, Italy ; Battaglino, C. ; Damiano, R. ; Nunnari, F. "'An Avatar-based Interface for the Italian Sign Language", Complex, Intelligent and Software Intensive Systems (CISIS), 2011 International Conference on,2011

13. Rakun, E. ; Fac. of Comput. Sci., Univ. of Indonesia, Depok, Indonesia ; Febrian Rachmadi, M. ; Andros ; Danniswara, K. ,"Spectral domain cross correlation function and generalized Learning Vector Quantization for recognizing and classifying Indonesian Sign Language", Advanced Computer Science and Information Systems (ICACSIS), 2012 International Conference on ,2012

14. Hatami, N. ; Dipt. di Autom. e Inf., Politec. di Torino, Turin, Italy ; Prinetto, P. ; Tiotto, G.,"Sign Language synthesis using hand motion acquisition", Design \& Test Symposium (EWDTS), 2010 East-West, 2010

15. Grif, M.G. ; Dept. of Autom. Control Syst., Novosibirsk State Tech. Univ., Novosibirsk, Russia ; Demyanenko, Y.A. ; Korolkova, O.O. ; Tsoy, Y.B. ,"Development of computer sign language translation technology for deaf people", Strategic Technology (IFOST), 2011 6th International Forum on (Volume:2 ), 2011

16. Eunseok Jeong; Dept. of Electr. \& Electron. Eng., Yonsei Univ., Seoul, South Korea ; Jaehong Lee; DaeEun Kim “ Finger-gesture Recognition Glove using Velostat (ICCAS 2011), Control, Automation and Systems (ICCAS), 2011 11th International Conference on,2011

17. Barczewska, K. ; Dept. of Autom. Control \& Biomed. Eng., AGH Univ. of Sci. \& Technol., Kraków, Poland ; Drozd, A., "Comparison of methods for hand gesture recognition based on Dynamic Time Warping algorithm", Computer Science and Information Systems (FedCSIS), 2013 Federated Conference on,2013

18. Majeau, L. ; PolyProject, Tech. Soc. of Polytech. of Montreal, Montreal, QC, Canada ; Borduas, J. ; Loranger, S. ; El-Iraki, Y. ,"Dataglove for consumer applications",Fibre and Optical Passive Components (WFOPC), 2011 7th Workshop on ,2011

19. Haoyum Xue ; Sch. of Eng. \& Design, Brunel Univ., Uxbridge, UK ; Shengfeng Qin ,"Mobile motion gesture design for deaf people", Automation and Computing (ICAC), 2011 17th International Conference on, 2011

20. Boulares, Mehrez; Research Laboratory of Technologies of Information and Communication \& Electrical Ingineering (LaTICE), Ecole Supérieure des Sciences et Techniques de Tunis, 5th Av. Taha Hussein, B.P. 56, Bab Mnara 1008, TUNISIA ; Jemni, Mohamed "Toward HMM based machine translation for ASL", Information and Communication Technology and Accessibility (ICTA), 2013 Fourth International Conference on ,2013

21. Mohandes, M. ; Dept. of Electr. Eng., King Fahd Univ. of Pet. \& Miner., Dhahran, Saudi Arabia ; Junzhao Liu ; Deriche, M., "A survey of image-based Arabic sign language recognition", Multi-Conference on Systems, Signals \& Devices (SSD), 2014 11th International, 2014

22. Dhahran 31261, Saudi Arabia (e-mail: mohandes@kfupm.edu.sa; mderiche@kfupm.edu.sa; liujunzhao@kfupm.edu.sa). ; Deriche, M. ; Liu, J. ,"Image-Based and Sensor-Based Approaches to Arabic Sign Language Recognition”,Human-Machine Systems, IEEE Transactions on (Volume:PP , Issue: 99 ),2014

23. Rakun, E. ; Fac. of Comput. Sci., Univ. Indonesia, Depok, Indonesia ; Andriani, M. ; Wiprayoga, I.W. ; Danniswara, K., "Combining depth image and skeleton data from Kinect for recognizing words in the sign system for Indonesian language (SIBI [Sistem Isyarat Bahasa Indonesia])" , Advanced Computer Science and Information Systems (ICACSIS), 2013 International Conference on, 2013

24. Shujjat Khan etal, Sign Language Analysis and Recognition: A Preliminary Investigation, 24th International Conference Image and Vision Computing New Zealand (IVCNZ 2009)

25. M N Sowjanya, S N Thimmaraju, A Comparison of Methods Used to Convert Sign Languages to Relative Formal Languages, JARDCS, Vol. 11, 01-Special Issue, 2019

26. M N Sowjanya, S N Thimmaraju, Multiple Hidden Neuron based model foraccurate ASL translation, JATZ, October 2020,

27. Teena Varmaetal, Sign Language Detection using Image Processing and Deep Learning, IRJET, Volume: 07

Issue:

11 , 2020. 